\title{
Pair Cascades and Deathlines in Magnetic Fields with Offset Polar Caps
}

\author{
Alice K. Harding* and Alex G. Muslimov ${ }^{\dagger}$ \\ *Astrophysics Science Division, NASA/Goddard Space Flight Center, Greenbelt, MD 20771 \\ ${ }^{\dagger}$ Universities Space Research Association/CRESST, Columbia, MD 21044
}

\begin{abstract}
We present results of electron-positron pair cascade simulations in a dipole magnetic field whose polar cap is offset from the dipole axis. In such a field geometry, the polar cap is displaced a small fraction of the neutron star radius from the star symmetry axis and the field line radius of curvature is modified. Using the modified parallel electric field near the offset polar cap, we simulate pair cascades to determine the pair deathlines and pair multiplicities as a function of the offset. We find that the pair multiplicity can change dramatically with a modest offset, with a significant increase on one side of the polar cap. Lower pair deathlines allow a larger fraction of the pulsar population, that include old and millisecond pulsars, to produce cascades with high multiplicity. The results have some important implications for pulsar particle production, highenergy emission and cosmic-ray contribution.
\end{abstract}

Keywords: Neutron stars:pulsars

PACS: $97.60 . \mathrm{Gb}, 97.60 . \mathrm{Jd}$

\section{INTRODUCTION}

Rotation-powered pulsars are thought to produce electron-positron pairs through electromagnetic cascades in their magnetospheres. Such pair cascades may occur both in the magnetic polar regions, where high energy photons radiated by accelerated particles undergo pair conversion in the strong near-surface magnetic fields [7] or in outer gaps, where high energy photons interact with thermal X-rays from the neutron star (NS) surface [6]. There has been a long-standing problem in understanding how long-period pulsars are able to produce coherent radio emission, thought to require electron-positron pairs [3] and standard pulsar models are not able to account for the operation of robust pair cascades in these aging pulsars. More recently, the discovery of pulsed gamma-ray emission from a large number of millisecond pulsars by the Large Area Telescope (LAT) on the Fermi Gamma-Ray Space Telescope [1] has revealed light curves that are best modeled by narrow radiation gaps in the outer magnetosphere [13, 2]. Such narrow gaps require screening of the accelerating electric field over most of the magnetosphere by a pair multiplicity that is orders of magnitude larger than standard models are able to produce [11].

This has motivated us to investigate the effect of a large-scale non-dipolar NS magnetic field geometry on pulsar pair cascades. Off-centered dipole-like magnetic fields seem to be prevalent among planets in the Solar System and in some stars. Recent modeling of X-ray pulse profiles from millisecond pulsars (MSPs) shows evidence for offset dipole fields or offset polar caps in PSR J0437-4715 [4] and PSR J0030+0451 [5]. Arons [3] argued that pair creation in long period $(P>1 \mathrm{~s})$ pulsars was difficult and that dis- 
tortions of polar field lines due to large-scale, non-dipolar surface fields of magnitude comparable to the dipole component, and of much smaller radius of curvature, could enable pair creation in older pulsars.

\section{MAGNETIC AND ELECTRIC FIELD OF A DISTORTED DIPOLE}

We use a simplified model of an asymmetric magnetic field of a pulsar by introducing an azimuthal asymmetry to the field lines of an originally symmetric dipole, such that the field lines over half of the polar cap (PC) have relatively smaller radius of curvature. Consequently, one side of the PC is larger and the PC is effectively shifted from the [star] center of symmetry. In magnetic spherical polar coordinates $(\theta, \phi)$ the magnetic field is assumed to have the following form:

$$
\mathbf{B} \approx \frac{B_{0}}{\eta^{3}}\left[\hat{\mathbf{r}} \cos [\theta(1+a)]+\frac{1}{2} \hat{\theta} \sin [\theta(1+a)]\right],
$$

where $\eta=r / R_{\mathrm{ns}}$ is the dimensionless radial coordinate in units of stellar radius, $a$ is the parameter characterizing the distortion of polar field lines. If the magnetic axis lies in the $\mathrm{x}-\mathrm{z}$ plane, $a=\varepsilon \cos \phi$.produces an effective offset of the $\mathrm{PC}$ in the $\mathrm{x}-\mathrm{z}$ plane and $a=\varepsilon \sin \phi$ an effective offset of the PC in the $y-z$ plane, where $0 \leq \varepsilon<1$. The effective shift of the PC on the NS surface is approximately, $\Delta r_{\mathrm{PC}} \simeq R_{\mathrm{ns}} \theta_{0}\left[1-\theta_{0}^{\varepsilon}\right]$, where $\theta_{0}=$ $\left(\Omega R_{\mathrm{ns}} / c\right)^{1 / 2}$ is the canonical half-angle of the PC, $\Omega$ is the pulsar rotation rate and $R_{\mathrm{ns}}$ is the NS radius. Since the effective offset of the PC is a fraction of the PC pening angle, it is a very small fraction of the stellar radius for normal pulsars and somewhat larger fraction for millisecond pulsars. Neglecting the static general-relativistic corrections, we can write the accelerating electric field, assuming the boundary conditions of spacecharge limited flow (SCLF) (cf. [10], as

$$
E_{\mid i} \approx-E_{0} \frac{x^{a}}{(1+a)^{2}}\left\{\frac{\kappa}{\eta^{4}}\left[3+a\left(\eta^{3}-1\right)\right] \cos \chi+\frac{3}{8} \frac{x^{a / 2}}{(1+a)} \frac{\theta_{0}}{\sqrt{\eta}} \xi \sin \chi \cos \phi\right\}\left(1-\xi^{2}\right),
$$

where, $E_{0}=(1 / 2)\left(\Omega R_{\mathrm{ns}} / c\right)^{2} B_{0}, \chi$ is the pulsar obliquity, $x=r / r_{L C}$, where $r_{L C}=c / \Omega$ and $\kappa \approx 0.15 I_{45} / R_{6}^{3}\left(I_{45}=I / 10^{45} \mathrm{~g} \cdot \mathrm{cm}^{2}, R_{6}=R_{\mathrm{ns}} / 10^{6} \mathrm{~cm}, I\right.$ is NS moment of inertia) is the parameter accounting for the general-relativistic frame dragging [10].

\section{PAIR CASCADE SIMULATION AND RESULTS}

Using the distorted field structure and accelerating electric field described above, we have simulated a full pair cascade above the pulsar polar cap to compare the pair multiplicity and pair death lines for different offset parameters $\varepsilon$. The full pair cascade simulation code, described in [7], has been combined with the acceleration, early cascade development and pair front formation used in [10]. The increase in pair multiplicity from a distorted dipole field can move many pulsars above the death line for robust pair creation. Figure 1 shows the death lines for pair creation by CR in the $P-\dot{P}$ diagram for 


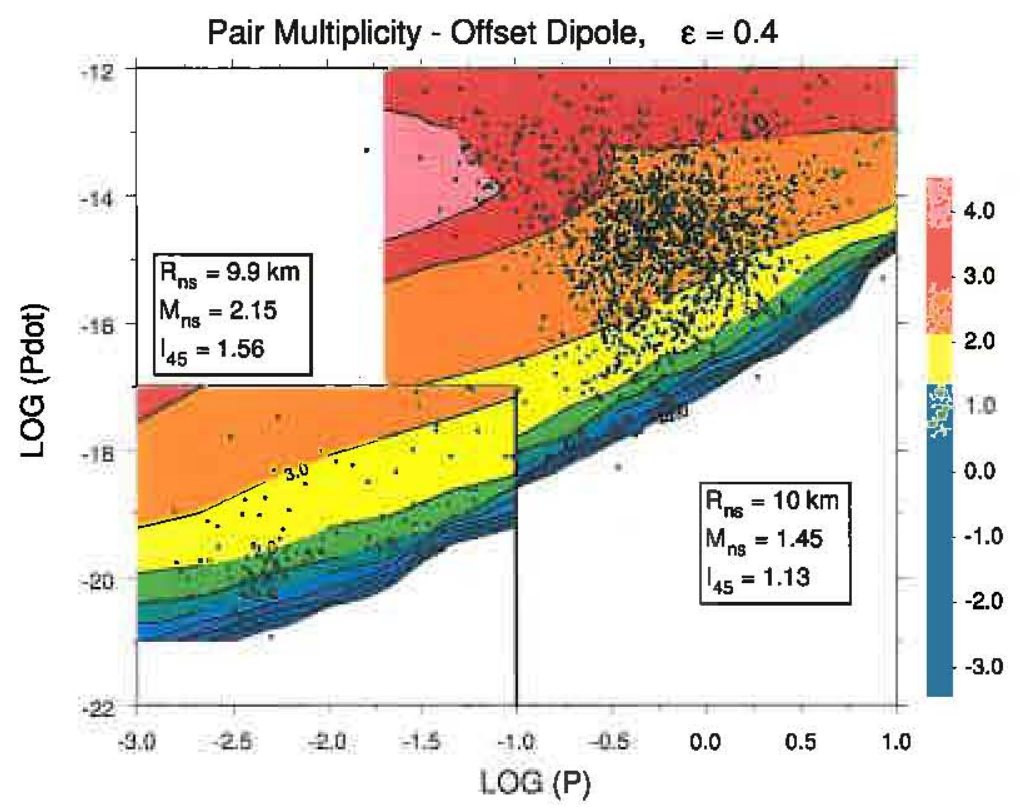

FIGURE 2. Log of pair multiplicity in the $P-\dot{P}$ plane for offset parameter $\varepsilon=0.4$.

which is by far the strongest effect. Since this effect is sensitive to effective offsets that are fractions of a PC radius, we show that many older pulsars and MSPs would be able to sustain robust pair cascades with dipole offsets that are small fractions of a stellar radius. Distortions of the dipole field could be intrinsic to the NS through asymmetries in the interior currents, present either from birth or as a result of spin-up or spin-down evolution. Distortion of the magnetic field by rotation or magnetospheric currents also produce offsets of the PC [8] by as much as $20-30 \%$ of the PC radius and this should be taken into account in PC acceleration and pair cascades.

\section{REFERENCES}

1. Abdo, A. A. et al. 2009 , Science, 325,848 .

2. Abdo, A. A. et al. 2010, ApJ, 712, 957

3. Arons, J. 1997, in Neutron Stars and Pulsars: Thirty Years after the Discovery, eds. N. Shibazaki, N. Kawai, S. Shibata and T. Kifune, Univ. Academy Press, Inc., Tokyo, Japan, p. 339.

4. Bogdanov, S. , Rybicki, G. B. \& Grindlay, J. E. 2007, ApJ, 670, 668.

5. Bogdanov, S. \& Grindlay, J. E. 2009, ApJ, 703, 2259.

6. Cheng, K. S., Ho, C., \& Ruderman, M. A. 1986, ApJ, 300, 500.

7. Daugherty, J. K. \& A. K. Harding 1982, ApJ, 252, 337.

8. Dyks, J. \& Harding, A. K. 2004, ApJ, 614, 869.

9. Friedman, J. L., Ipser, J. R., \& Parker, L. 1986, ApJ, 304, 115.

10. Harding, A. K., \& Muslimov, A. G. 1998, ApJ, 508, 328.

11. Harding, A. K., \& Muslimov, A. G. 2002, ApJ, 568, 862.

12. Lattimer, J. M. \& Prakash, M. 2007, Physics Reports, 442, 109.

13. Venter, C.; Harding, A. K.; Guillemot, L. 2009, ApJ, 707, 800. 